Full Length Article

\title{
Combustion states distinction of the methane/oxygen laminar co-flow diffusion flame at high pressure
}

\author{
Yifei $\mathrm{Ge}^{\mathrm{a}, \mathrm{b}}$, Sen $\mathrm{Li}^{\mathrm{a}, *}$, Xiaolin $\mathrm{Wei}^{\mathrm{a}}$ \\ ${ }^{a}$ State Key Laboratory of High Temperature Gas Dynamics, Institute of Mechanics, Chinese Academy of Sciences, Beijing 100190, China \\ ${ }^{\mathrm{b}}$ University of Chinese Academy of Sciences, Beijing 100049, China
}

\section{A R T I C L E I N F O}

\section{Keywords:}

Diffusion flame

Co-flow

Laminar

Pulsation

High pressure

\begin{abstract}
A B S T R A C T
Compared to turbulent combustion in practical combustion devices, a co-flow laminar diffusion flame is the simplest configuration from which the interactions between flow field and reactions can be readily studied. High pressure combustion experiments on the methane/oxygen laminar co-flow diffusion flame are conducted to distinguish combustion states from 0.2 to $4 \mathrm{MPa}$. The transition combustion state between the steady state and periodical pulsation combustion state is studied quantitatively. The results obtained using a Schlieren device show that the different formation heights of the vortices generated in the jet flame shear layer lead to different combustion states. Based on the combustion pressure and Froude number at the burner exit, the upper and lower limits for the transition combustion state are proposed as $F r_{\text {cr }}=9.8 \times 10^{-4}\left(p_{\mathrm{r}}-0.012\right)^{-1.87} \pm 4.0 \times 10^{-5}\left(p_{\mathrm{r}}+0.026\right)^{-2.48}$, respectively. The flame is in the steady

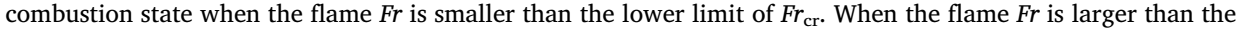
upper limit of $F r_{\mathrm{cr}}$, pulsation combustion takes place. For the pulsation combustion state, the relationship between the Strouhal number and Froude number is established as $S t=0.29 \times \mathrm{Fr}^{-0.65}$.
\end{abstract}

\section{Introduction}

In a methane/oxygen liquid rocket motor, flame instability at high pressure ( $>2 \mathrm{MPa}$ ) gives rise to local pulsation in the pressure and temperature fields, leading to inadequate burning that in turn leads to mechanical failure or even explosions [1,2]. Flame instability, especially pulsation, is associated with the interaction between the vortices in the flow of the flame. Turbulent combustion commonly occurs in practical combustion devices. The experimental measurements of turbulent combustion are restricted by the high level of intermittency due to turbulent motion and a relatively short residence time [3,4]. Turbulent combustion can be regarded as a combination of microlaminar combustion with turbulence mass transfer, and computational models such as the flamelet model are based on this theory [5]. For the turbulent flow in a rocket chamber, the magnitude of the Taylor microscale Reynolds number $R e_{\lambda}$ is usually 10-100. Thus, the combustion features can be studied using a laminar flame with the same magnitude of $R$. Compared to the devices used for turbulent combustion, the laminar co-flow diffusion flame experimental device has a simple configuration with controllable boundary conditions from which the interactions between the flow field and the reactions can be fundamentally modified and studied.
As a typical hydrocarbon fuel, methane is widely used in practical conditions. Many researchers have focused on the methane/air flame, while little information is available on the high-pressure methane/ oxygen characteristics in the literature [3]. Miller and Maahs [6] studied the methane/air steady flame in a high-pressure chamber. Their study showed that with increasing pressure, the reaction mechanism of flame changes, resulting in the presence of increasing amounts of carbon in the flame. As a result, the flame shape undergoes a remarkable change with increasing pressure. A similar result was reported by Karatas, Intasopa and Gülder [7] in their studies of the hydrocarbon/ oxygen flame at high pressure. Their work showed that increasing the combustion pressure effectively enhances the combustion intensity.

Pulsation features of the laminar diffusion flame have also been widely observed. Kimura [8] and Durox et al. [9] studied the stability of laminar diffusion flame, results shows that the flame flickering has relation with flame height, that the minimum height of flame is necessary to trigger the flickering. Similar phenomenon had been found by Zhang and Fang [10]. They studied the methane/air flame shape induced by oxygen concentration and air-velocity under microgravity conditions. The increasing of oxygen concentration in co-flow air decreases the flame height, which, in turns stabilized the flame. Lewis [11] studied the vortices structure of unsteady diffusion flame. The

\footnotetext{
* Corresponding author.

E-mail address: lisen@imech.ac.cn (S. Li).
} 


$\begin{array}{ll}\text { Nomenclature } \\ F r & \text { Froude number [-] } \\ S t & \text { Strouhal number [-] } \\ p & \text { Pressure [MPa] } \\ R e_{\lambda} & \text { Taylor microscale Reynolds number }[-] \\ u & \text { Characteristic velocity }[\mathrm{m} / \mathrm{s}] \\ l & \text { Characteristic length }[\mathrm{m}] \\ g & \text { Gravity acceleration }\left[\mathrm{m} / \mathrm{s}^{2}\right] \\ t & \text { Time }[\mathrm{s}]\end{array}$

velocity distribution near flame shear layer demonstrated that the flame flickering phenomenon is induced by vortices structure generated out of flame reaction zone. Charwath et al. [12] and Papadopoulos et al. [13] studied flame shape changing process during flame pulsation using radicals and soot distribution in methane/air diffusion oscillating flame. At the same time, theoretical analysis on flame pulsation process are provided by many researchers $[8,9,14]$. Darabkhani et al. [15] suggested that flame pulsation modes vary for different fuels. Three combustion states of the methane diffusion flame have been found by numerous researchers, namely, the steady, transition and pulsation combustion states. Hegde, Zhou and Bahadori [16] suggested that pulsation combustion initiation is not continuous and shows an intermittent behaviour, where a transition state is found between steady combustion and continuous pulsation combustion. For the laminar coflow diffusion flame, the Kelvin-Helmholtz instability is considered the leading origin of flame pulsation, which has important implications in numerous applications involving non-reacting shear layers as well as reacting mixing layers [17]. However, most of the flame pulsation studies have been dedicated to the continuous pulsation combustion phenomenon, while the studies of transition pulsation combustion have been rare [18], and the critical conditions for the transition combustion state has not been studied in detail.

In this study, the stability characteristics of a laminar co-flow methane/oxygen diffusion flame were investigated at $p=0.2-4.0 \mathrm{MPa}$, the critical conditions for the three combustion states were measured, and the flame pulsation mechanism was determined using the Schlieren method.

\section{Experimental facility and analysis method}

\subsection{Experimental facility}

The experimental system is shown in Fig. 1 and includes the pressure combustion chamber and the control and measurement system. Three thermally based mass flow metres (Sevenstar D07-11C) were used to control the flow rates of methane, oxygen and nitrogen, and their maximum errors were less than $5 \%$ at $p<5.0 \mathrm{MPa}$. In the experiment, the methane mass flow rate was varied from 0.805 to $2.440 \mathrm{mg} / \mathrm{s}$. A pressure transducer was installed on the top of the pressure chamber for monitoring the pressure. Experiments were conducted at 0.2, 0.3, 0.5, 0.7, 1.0, 1.5, 2.0, 2.5, 3.0 and 4.0 MPa.

The structure of the high-pressure combustion chamber is shown in Fig. 2. The design of the high-pressure combustion chamber is described in Ref. [3]. The inner diameter and height of the combustion chamber are $226 \mathrm{~mm}$ and $600 \mathrm{~mm}$, respectively. Three view ports covered with quartz glasses were set on the chamber, providing sights of $0^{\circ}, 90^{\circ}$ and $180^{\circ}$ for optical observation. A nitrogen inlet on the bottom was used to pressurize the chamber. The water mist coagulating on the view-port glasses was removed by high-pressure nitrogen gas. A spiral cooling pipe was placed above the burner to condense the water vapour of the flue gas in the chamber.

A co-flow burner (Fig. 1b) was installed in the centre of the highpressure combustion chamber. Methane concentrically issued from the

$\begin{array}{ll}f_{t} & \text { Pulsation frequency [Hz] } \\ f_{s} & \text { Sampling frequency [Hz] } \\ N_{F F T} & \text { Number of samples for FFT [-] } \\ \Delta R_{f} & \text { Frequency resolution [Hz] } \\ \text { Subscripts } \\ \text { cr } & \text { Critical [-] } \\ \mathrm{r} & \text { Relative [-] } \\ \text { cent } & \text { Center line/Average [-] } \\ \text { crmax } & \text { Upper critical limit [-] } \\ \text { crmin } & \text { Lower critical limit [-] }\end{array}$

burner port with an internal diameter of $3.01 \mathrm{~mm}$ into the oxygen stream. This burner port is encompassed by a chimney that has an internal diameter of $30 \mathrm{~mm}$ and is $80 \mathrm{~mm}$ higher than the burner port so that the combustion is conducted in an oxygen atmosphere. Glass beads were filled into the chimney to smooth the oxygen stream. To observe the co-flow flame, three windows covered with quartz glasses were set on the chimney.

\subsection{Measurement method}

Flame images were obtained using an SLR camera (Nikon D90, image resolution $4288 \times 2848$ ) that was mounted with a Nikkor $105 \mathrm{~mm}$ fixed-focus lens. The frequency and magnitude of flame pulsation were studied using a photomultiplier (ET Enterprises). The diffusion flame had a two-zone structure under a high-pressure environment, with an inner zone luminous in yellow and an outer zone in blue [3]. In the experiment, the variation magnitude of flame outer zone was larger than that of the inner zone during pulsation, and a short-pass filter (Thorlabs FES0500, cut-off wave length $500 \mathrm{~nm}$ ) was placed in front of the photomultiplier to capture the light from the outer zone and transform it into a voltage signal. The voltage signal was input into a digital oscilloscope (Tektronix DPO 4032) to be recorded. The sampling frequency and time of oscilloscope are $1000 \mathrm{~Hz}$ and $10 \mathrm{~s}$ respectively. In the experiment, vertical scale was set at $200 \mathrm{mV} / \mathrm{div}$, resolution of voltage signal is $62.5 \mu \mathrm{V}$, typical random noise is less than $6.62 \mathrm{mV}$.

Based on the luminous intensity variation data, the flame pulsation frequency was obtained using the fast Fourier transform (FFT) algorithm [9,21-23], and different combustion states of the flame were distinguished by the short-time Fourier transform (STFT) algorithm $[19,20]$.

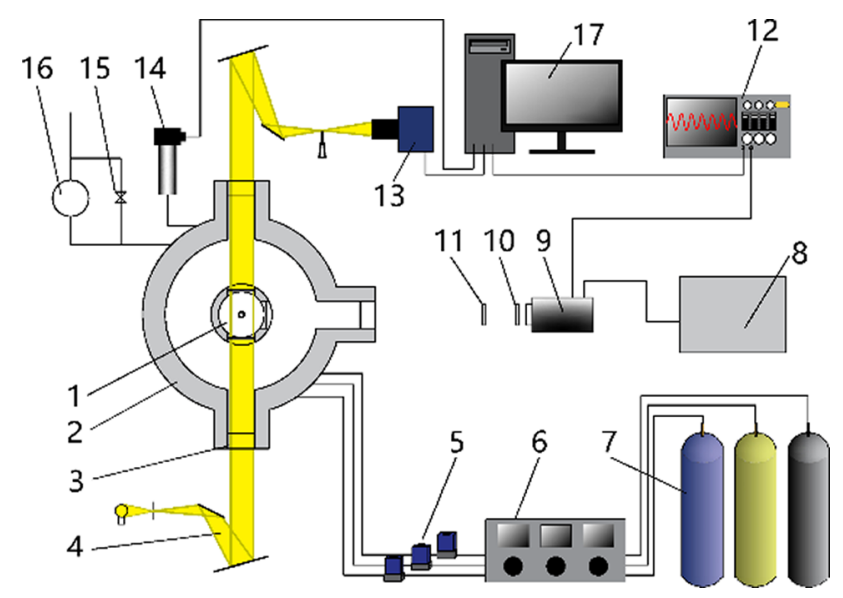

Fig. 1. Experimental control and measurement system. (1) Co-flow burner; (2) Pressure chamber, (3) Viewport; (4) Schlieren Light Path; (5) Mass flow metres; (6) Valve group; (7) Gas cylinders; (8) DC power source; (9) Photomultiplier; (10) Shortpass filter; (11) Neutral density filter; (12) Oscilloscope; (13) High speed camera; (14) Pressure transducer; (15) Safety valve; (16) Back pressure valve. 


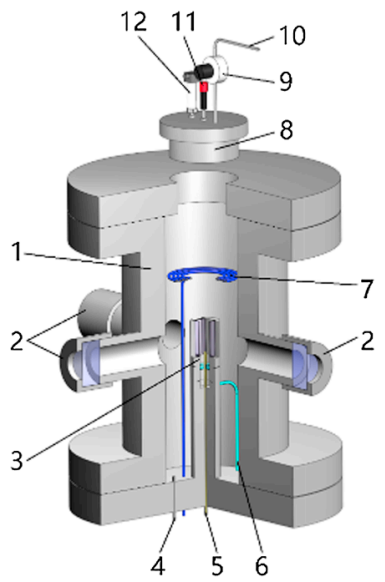

(a) Combustion chamber

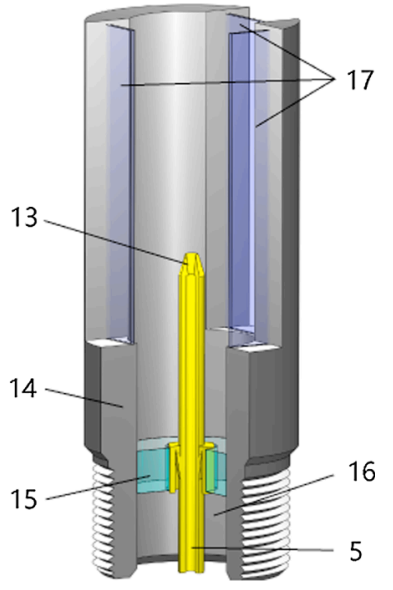

(b) Co-flow burner
Fig. 2. High-pressure combustion chamber and burner. (1) Chamber wall; (2) Viewports; (3) Co-flow burner; (4) Nitrogen inlet; (5) Methane inlet; (6) Oxygen inlet; (7) Colling pipe; (8) Chamber lid; (9) Back pressure valve; (10) Exhaust pipe; (11) Safety valve; (12) Pressure transducer; (13) Burner port; (14) Chimney; (15) Glass beads; (16) Oxygen tunnel; (17) Quartz glasses.

To analyse the flow field of the diffusion flame, the Schlieren method was used. The Schlieren device consisted of a sodium lamp, a slit, four concave mirrors, a knife-edge spatial filter and a high-speed camera. The light path of the Schlieren device is shown in Fig. 1. Flow field evolution during the experiment was obtained with a high-speed camera (Mikrotron MotionBLITZ EoSens Cube 7, image resolution $496 \times 1316$ ), with the frame rate set to $1000 \mathrm{fps}$.

It is commonly considered that self-induced flame pulsation is induced by Kelvin-Helmholtz instability in flow field at elevated pressure. Thus, flow parameter Froude number $(F r)$ is used in this study to describe flame flow field. $F r$ is defined as the ratio of the inertia force and the buoyancy force and is given by:

$F r=\frac{u^{2}}{l \cdot g}$

where $u$ is the average methane flow speed at the burner port, $\mathrm{m} / \mathrm{s} ; g$ is the gravity acceleration, $\mathrm{m} / \mathrm{s}^{2}$; and $l$ is the characteristic length (here, $l$ is the diameter of the burner port), $\mathrm{m}$.

\section{Results and discussion}

\subsection{Differences between the flame states}

Three periods of flame luminous intensity histories recorded using an oscilloscope were chosen as examples and are shown in Fig. 3. Significant differences are observed between these data logs. In Fig. 3a $\left(F r=0.98 \times 10^{-3}\right)$, the data $\log$ is smooth, with only a slight vibration. No pulsation appears in this state, and the flame is in the steady combustion state. In Fig. $3 \mathrm{~b}\left(\mathrm{Fr}=2.79 \times 10^{-3}\right)$, with the growth in the Froude number, intermittent pulsation appears in the data log, indicating that the combustion is no longer steady. In this state, the regular pulsation behaviour did not form yet; thus, the flame is in the transition combustion state [16]. The experiments showed that the proportion of the pulsation segment will change with the variation in the Froude number of the flame, and, once the intermittent pulsation appears, growth in $\mathrm{Fr}$ leads to a larger proportion of the pulsation segment. As shown in Fig. 3c $\left(F r=7.83 \times 10^{-3}\right)$, for conditions of larger $F r$, the proportion of the pulsation segment is 1 , showing that pulsation combustion has been formed. The pulsation amplitude in Fig. $3 \mathrm{c}$ is larger than that for the transition combustion state shown in Fig. 3b. With the increasing Fr number, the steady flame (Fig. 3a) changes to the pulsation flame (Fig. 3c), while the curve in Fig. 3b shows the transition state between the steady flame and the pulsation flame.

To obtain the frequency features of the flame, the recorded luminous intensity histories were processed using the FFT method [21-23]. Fig. 4 shows the frequency-magnitude spectra at $3.0 \mathrm{MPa}$, for the same Froude numbers as those in Fig. 3. For the steady combustion state (Fig. 4a), the spectrum curve is flat in the high frequency region. The frequency of the fluctuation is less than $5 \mathrm{~Hz}$ and is due to unavoidable random comprehensive factors of the experimental system (system resonance, oscilloscope random noise and other factors). For the pulsation combustion state (Fig. 4c), the fluctuation power peak is observed at $34.4 \mathrm{~Hz}$, and the first-order harmonic power peak at approximately $69 \mathrm{~Hz}$ is also observed, indicating that pulsation combustion appears at these conditions [24,25]. As mentioned above, the sampling frequency $f_{s}=1000 \mathrm{~Hz}$, the sampling time is $10 \mathrm{~s}$, and the number of samples for FFT processing is $N_{\mathrm{FFT}}=10000$. The frequency resolution $\Delta R_{f}$ for FFT method is:

$\Delta R_{f}=f_{s} / N_{\mathrm{FFT}}$

Thus $\Delta R_{f}=0.1 \mathrm{~Hz}$. For pulsation flame at $\mathrm{Fr}=7.83 \times 10^{-3}, 3 \mathrm{MPa}$, FFT result shows the flame pulsation frequency is $34.4 \mathrm{~Hz}$. In the transition combustion state (Fig. 4b), a faint power peak with a minor magnitude occurs at approximately $39 \mathrm{~Hz}$, and the spectrum line is obviously different from those in Fig. $4 \mathrm{a}$ and c. For different combustion pressure, experiment results show that the spectrum curves in the same combustion states are similar, as shown in Figs. 4 and 5 (where Fig. 5a are curves for 1.0 MPa, and Fig. $5 \mathrm{~b}$ are for 4.0 MPa).

As seen from the above analysis, the methane/oxygen diffusion flame can be found in three combustion states depending on the changes in the flow conditions (i.e. Froude number). Which means the transition from steady combustion to pulsation combustion is not happened immediately [16], where a transition combustion state is found. In this case, it is essential to find the initial and terminal points of the transition combustion state, which correspond to the critical conditions that distinguish among the three combustion states. Spectrums in Fig. 6 are located near the initial and terminal points of the transition combustion state, where Fig. $6 \mathrm{a}$ is for steady combustion state, Fig. $6 \mathrm{~b}$ and $\mathrm{c}$ are for transition combustion state, Fig. $6 \mathrm{~d}$ is for pulsation combustion state. It can be seen that the difference between Fig. $6 \mathrm{a}$ and $\mathrm{b}$ is minor, as well as Fig. $6 \mathrm{c}$ and d. To find the initial and terminal points of the transition combustion state, it is necessary to involve a method that combines time-dependent and frequency features, and the short-time Fourier transform (STFT) method was used for such analysis in this work.

The STFT method divides the long-time data log into shorter

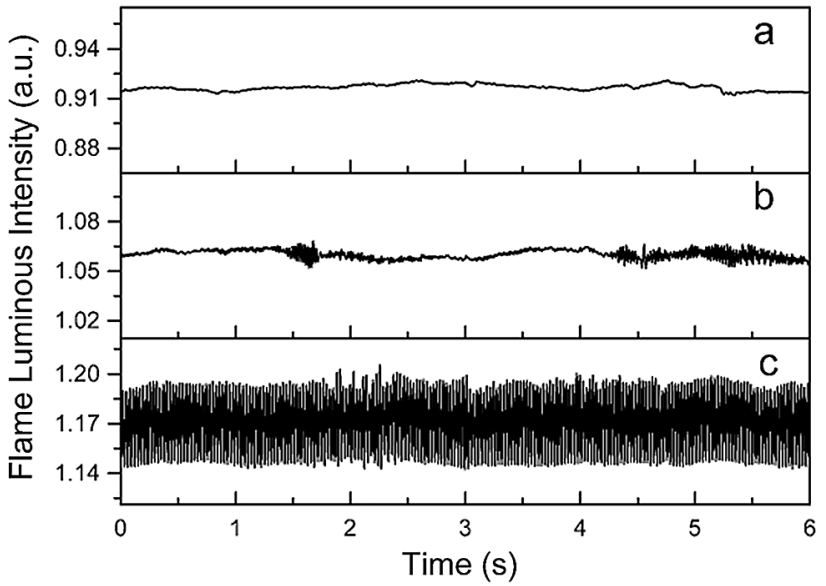

Fig. 3. Flame luminous intensity history at $3.0 \mathrm{MPa}$. (a) $\mathrm{Fr}=0.98 \times 10^{-3}$, (b) $\mathrm{Fr}=2.79 \times 10^{-3}$, (c) $\mathrm{Fr}=7.83 \times 10^{-3}$. 


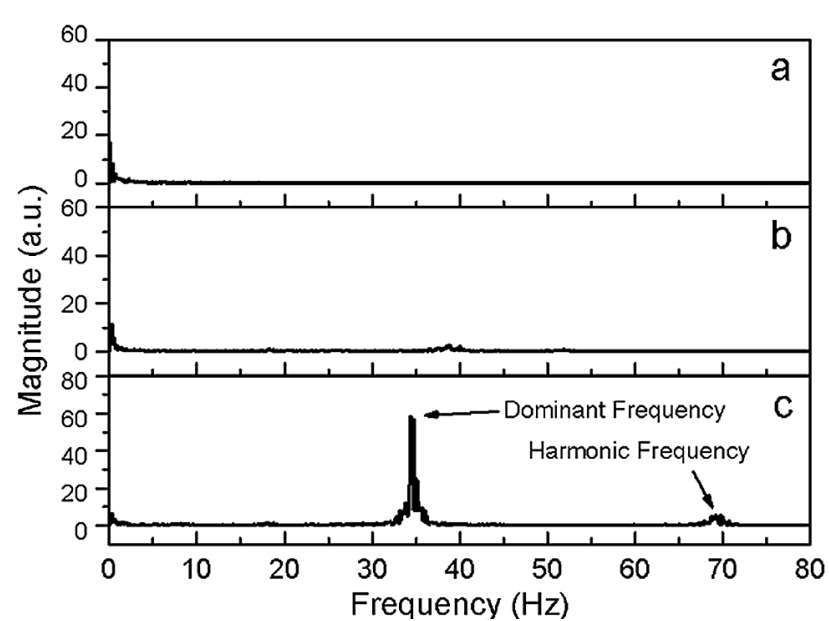

Fig. 4. Flame luminous intensity FFT frequency-magnitude spectrums at 3.0 MPa. (a) $\mathrm{Fr}=0.98 \times 10^{-3}$, (b) $\mathrm{Fr}=2.79 \times 10^{-3}$, (c) $\mathrm{Fr}=7.83 \times 10^{-3}$.

segments (or windows) with equal length and then computes the Fourier transform separately for each short segment. Thus, the frequency and phase content of the local sections are determined over time $[19,20]$. In this study, the window length was set to 256 out of 10,000 data points (1000 points per second), and the processing results are shown in Fig. 7. In these patterns, the fluctuation amplitude in $\mathrm{dB}$ is coloured from red (maximum $-1 \mathrm{~dB}$ ) to purple (minimum $-130 \mathrm{~dB}$ ).

Since the power supply frequency is $50 \mathrm{~Hz}$, the amplitude noise is observed at $50 \mathrm{~Hz}$ and $100 \mathrm{~Hz}$ (first harmonic frequency of the $50 \mathrm{~Hz}$ noise) in Fig. 7. At $\mathrm{Fr}=0.98 \times 10^{-3}$ (Fig. 7a), high dB flame fluctuation occurs in the low frequency region (noise band, close to $0 \mathrm{~Hz}$ ), and this combustion state is named the steady combustion state. With increasing $\mathrm{Fr}$ number, a high dB power peak drifts in the vicinity of $40 \mathrm{~Hz}$ at $F r=2.79 \times 10^{-3}$ (Fig. $7 \mathrm{~b}$ ). However, this power peak is not continuous; rather, some breaks appear in the time-frequency pattern, indicating that periodic pulsation combustion is not formed. This combustion state is named the transition combustion state. The emergence of a power peak deviating from the noise band indicates the initial appearance of the transition combustion state. By further increasing the Fr number to $\mathrm{Fr}=7.83 \times 10^{-3}$ (Fig. 7c), the amplitude power peak is sustained near $35 \mathrm{~Hz}$, and the harmonic power peak is found in the higher frequency region. In this case, periodic pulsation combustion is formed, and this combustion state is named the pulsation combustion state.

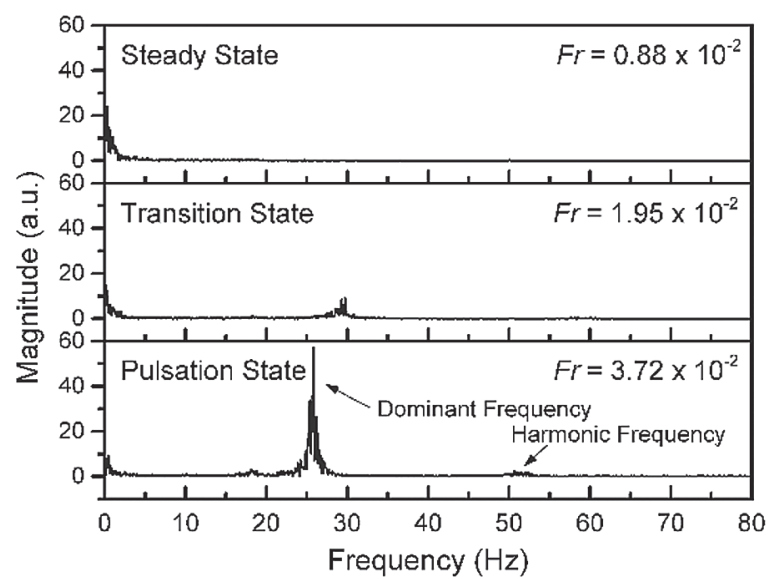

(a) Spectrum curves at $1.0 \mathrm{MPa}$

\subsection{Flow field of different combustion states}

To further analyse the features of the flame pulsation phenomenon with elevated pressure, the Schlieren method was used to study the flame flow field. Gas type, temperature difference and density difference lead to differences the in refractive index. A Schlieren device can capture this difference in the flame envelope and then obtain the details for the flow field pulse.

A comparison between the Schlieren image and flame photograph is shown in Fig. 8. The flame photograph was captured by an SLR camera (exposure of $1 / 3000$ s, Fig. 8a) and shows the typical two zone structure of the methane flame under elevated pressure [3], with an inner yellow zone and an outer blue zone. Furthermore, in Fig. 8b, since the external light source is necessary in the Schlieren measurements, the outer zone of the flame where the luminous intensity is weak can hardly be observed in the Schlieren image (exposure of $1 / 2000$ s). By contrast, the inner zone with greater luminous intensity is distinctly shown in the image. The boundary of the burnt gas and the gas heated by the flame, which is the interface between the high temperature gas and the colder ambient, is also distinctly visible in Fig. 8b. For the steady combustion state, this interface remains straight, and the flame flow field remains constant.

For the pulsation combustion state, significate differences can be found in both the SLR photographs and Schlieren images. The images in Fig. 9a and $9 \mathrm{~b}$ were obtained simultaneously. In Fig. 9a, the flame tip is separating from the flame body, and a vortex ring can be vaguely observed below the separating flame tip. In Fig. 9b, at the same height as that in Fig. 9a, this vortex ring (first vortex ring) under the flame tip can be clearly observed. In addition to this vortex ring, another vortex ring (second vortex ring) is clearly observed at a lower position. Fig. 9a shows that this lower vortex ring is located outside the outer zone of the flame. At the top of Fig. 9b, a large disturbance zone appears above these vortices.

The evolution of the vortices is shown in Fig. 10. The pulsation frequency for the flame at $3 \mathrm{MPa}$ and with $\mathrm{Fr}=7.83 \times 10^{-3}$ is $34.4 \mathrm{~Hz}$ (as obtained by FFT analysis described above); in other words, the flame pulsation period for these conditions is approximately $29.1 \mathrm{~ms}$. Fig. 10 shows nine consecutive Schlieren images for two periods with intervals of $7 \mathrm{~ms}$, together with the flame luminous intensity history at the same time. The trace of a vortex ring is marked by black arrows in the Schlieren images. At $t=0 \mathrm{~ms}$, the flame luminous intensity reaches the minimum value, and the height of the inner flame is also the smallest. The vortex ring is not generated yet. With the expansion of the high temperature interface $(t=7 \mathrm{~ms}$ and $14 \mathrm{~ms})$, the vortex ring emerges at $t=21 \mathrm{~ms}$ and then floats down steam and is finally

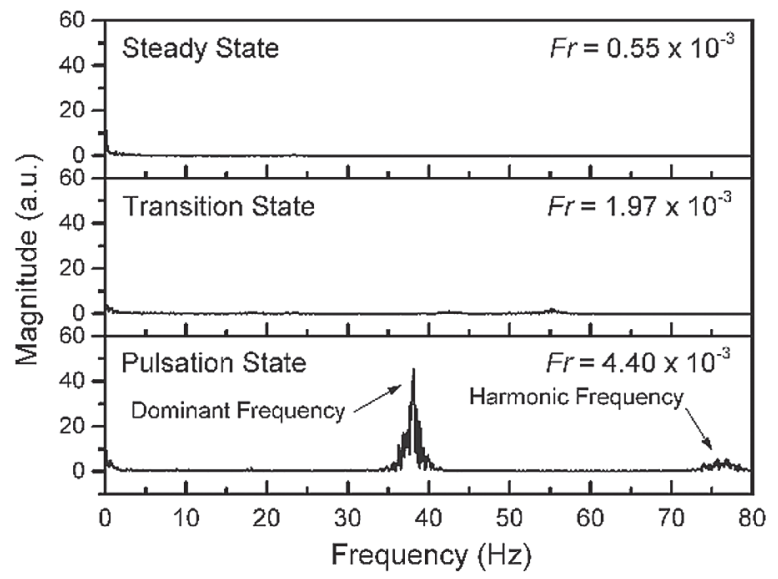

(b) Spectrum curves at $4.0 \mathrm{MPa}$

Fig. 5. Flame luminous intensity FFT frequency-magnitude spectrums at different pressure. 

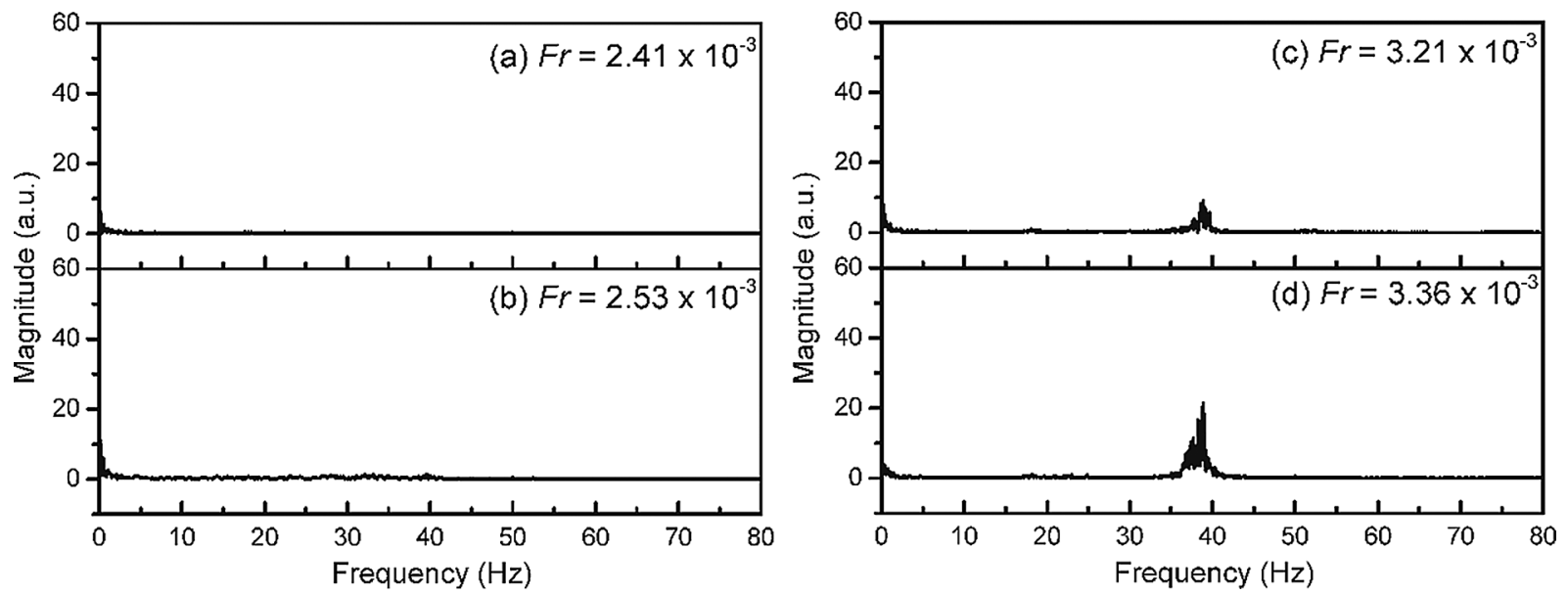

Fig. 6. Flame luminous intensity FFT spectrums at different $\operatorname{Fr}$ (3.0 MPa).

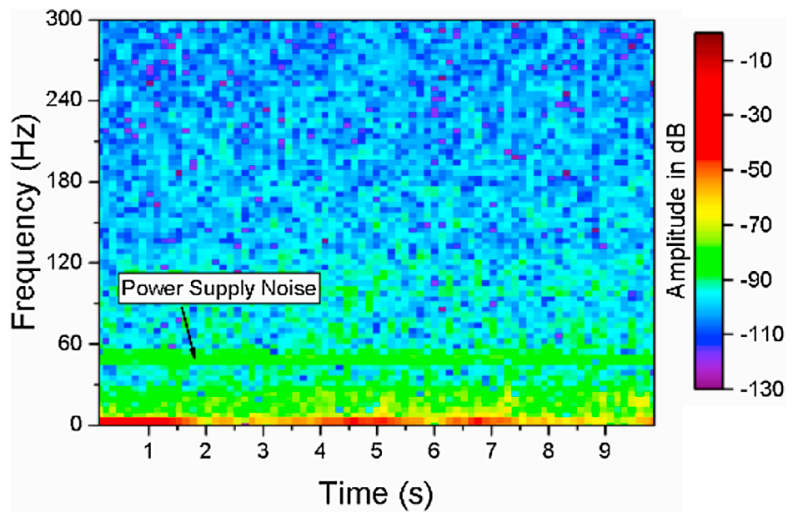

(a) $F r=0.98 \times 10^{-3}$

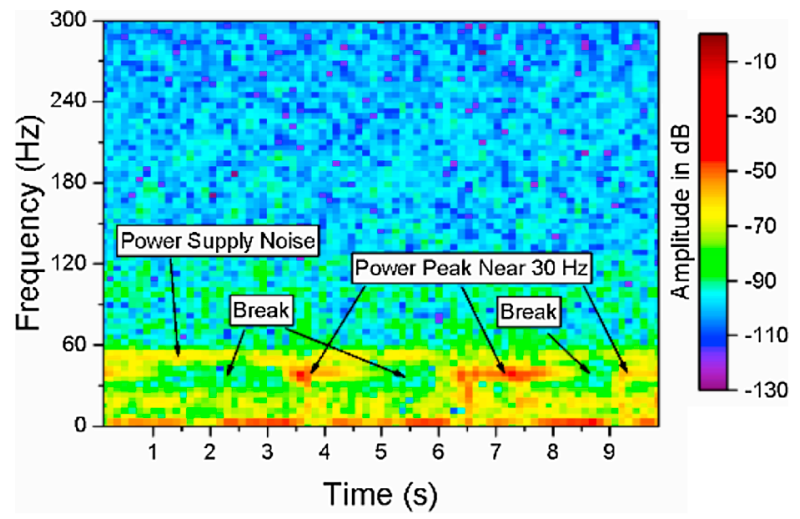

(b) $F r=2.79 \times 10^{-3}$

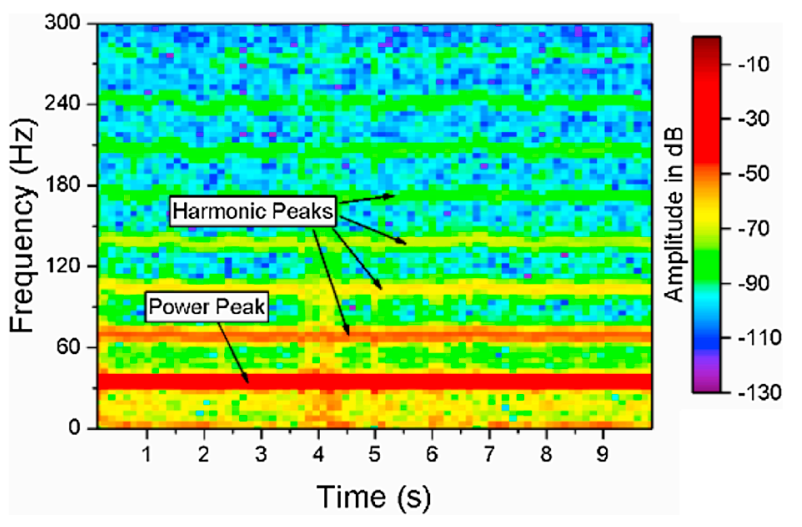

(c) $F r=7.83 \times 10^{-3}$

Fig. 7. Flame luminous intensity STFT time-frequency patterns at $3.0 \mathrm{MPa}$.

dissipated in the large disturbance zone. Then, at $t=28 \mathrm{~ms}$, a new vortex ring is ready to form (marked with white arrows).

As found from the examination of Fig. 9, the vortex ring emerges outside the flame reaction zone and floats outside the flame surface. This result means that these vortices were generated in the shear layer induced by the jet flame [11]. For the pulsation combustion state, the position of the vortices generation is essentially constant. The inflexional velocity profile in flame shear layer (as shown in Fig. 10) is induced by velocity gradient distribution caused by density difference near the shear layer. Therefore, the flow is unstable, and vortices generate periodically at the same position in the sense of KelvinHelmholtz. The boundary between burnt gas and co-flow oxygen is legible, and the flow is laminar around the flame plume. With the emergence and dissipation of these vortices, the flame reaction zone is entrained and deformed periodically together with the flame luminous intensity. As a result, the flame pulsation frequency is constant, as shown in Figs. 4c and 7c.

By contrast, for the transition combustion state, the vortex ring generation position shows large variation. The images in Fig. 11 are selected at the specific moments when the flame luminous intensity reaches the local minimum value. Noticeable differences in the vortex position are observed through the comparison of these images. For a higher generation position (Fig. 11a, for example), the flame reaction zone was not affected by the vortices, and the flame was steady 


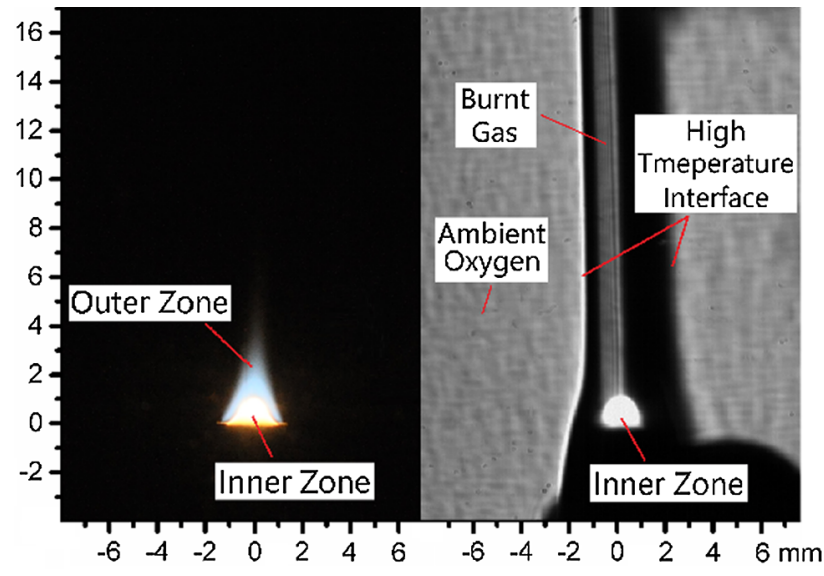

(a)

(b)

Fig. 8. Flame images of the steady combustion state (3 MPa, $F r=0.98 \times 10^{-3}$ ). (a) SLR photograph (b) Schlieren image.

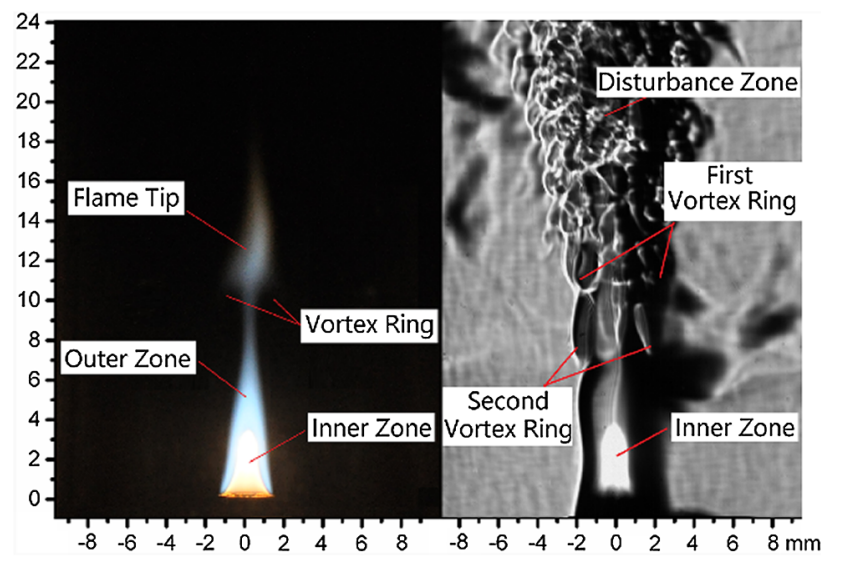

(a)

(b)

Fig. 9. Flame image of the pulsation combustion state (3 MPa, $F r=7.83 \times 10^{-3}$ ) (a) SLR photograph (b) Schlieren image.

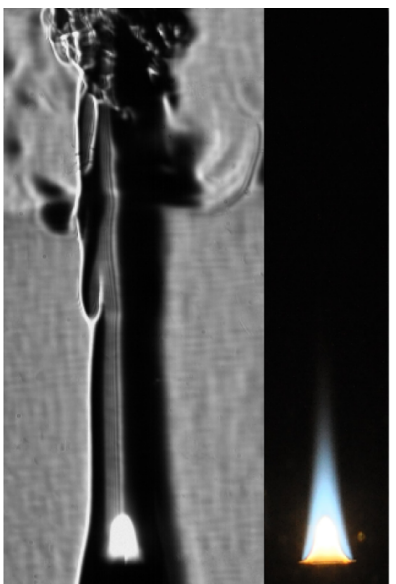

(a) $\mathrm{t}=0.010 \mathrm{~s}$

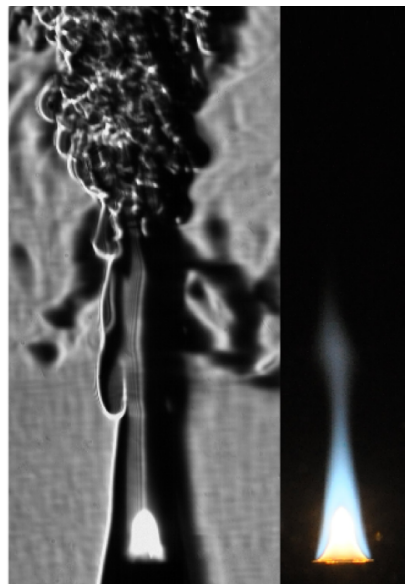

(b) $t=5.313 \mathrm{~s}$
Fig. 11. Flame images for the transition combustion state with minimum flame luminous intensity $\left(3 \mathrm{MPa}, \mathrm{Fr}=2.79 \times 10^{-3}\right)$.

temporarily (leading to the breaks observed in Fig. 7b). The flame starts to flicker only when the vortices were generated at a lower position (Fig. 11b), and the flame reaction zone is entrained by vortices.

The results show that the flame pulsation phenomenon was caused by periodical generation of vortices in the shear layer outside the fuel jet mixing layer due to buoyancy-driven Kelvin-Helmholtz instability. Kimura [8] and Durox et al. [9] found that the minimum height of flame is necessary to trigger the flickering. For a low flame Froude number, the flame height is relatively low, the flow field around the flame is stable, and either no vortices are generated, or the vortices are generated high above the flame reaction zone. When the flame Fr increases, flame height increases, while the height of the vortex generation position is decreased. When the height is sufficiently low so that the flame reaction zone is affected by the vortices, the flame starts to flicker. In a specific interval, the vortices generation height is flexible, leading to the flame alternating between the steady and flickering. With a further increase in the flame $F r$, flame height further increases, the vortices generation position becomes lower and is nearly invariable, leading to the periodic pulsation flame.

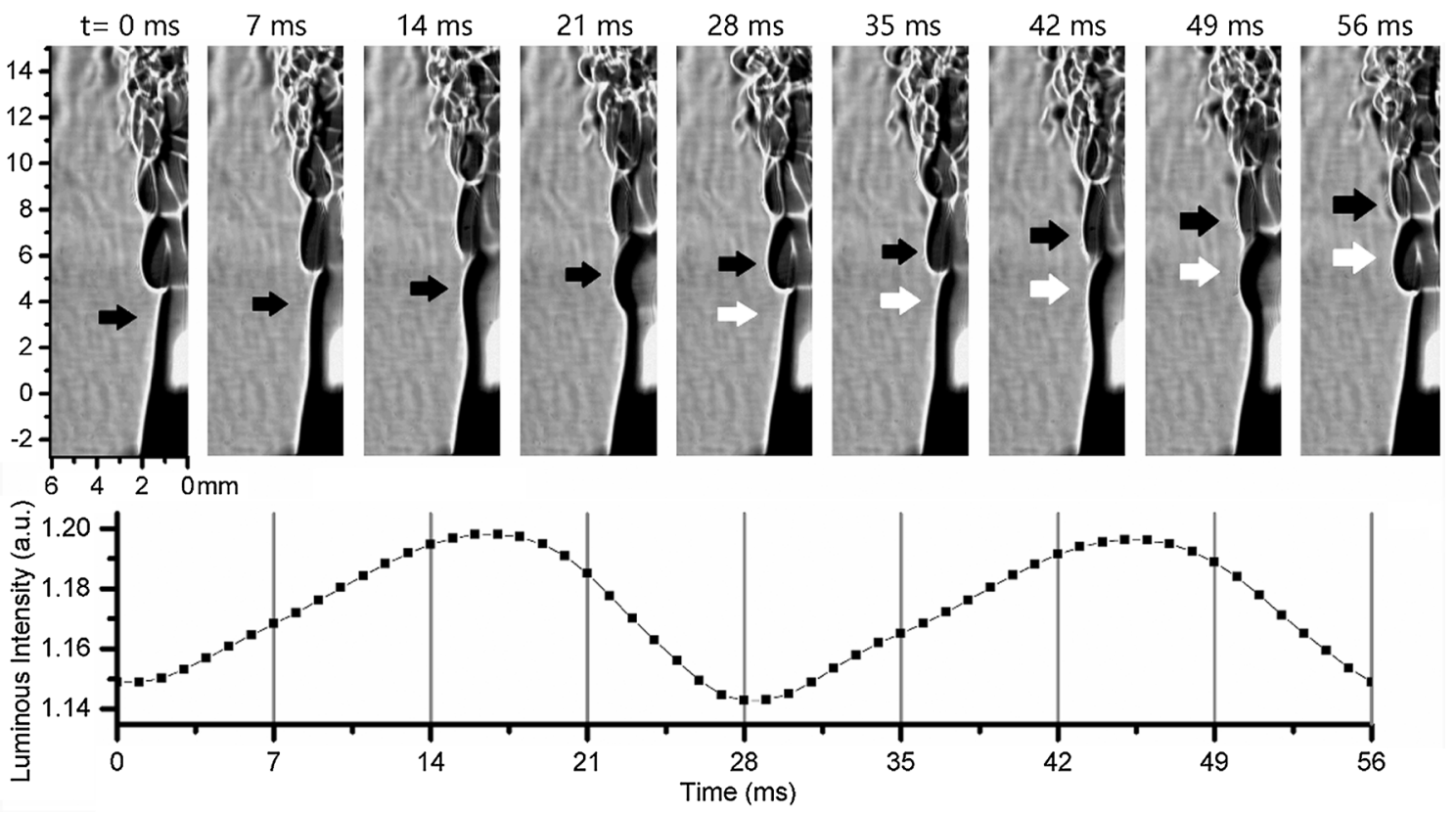

Fig. 10. Flow field evolution of the pulsation combustion state $\left(3 \mathrm{MPa}, \mathrm{Fr}=7.83 \times 10^{-3}\right)$. 


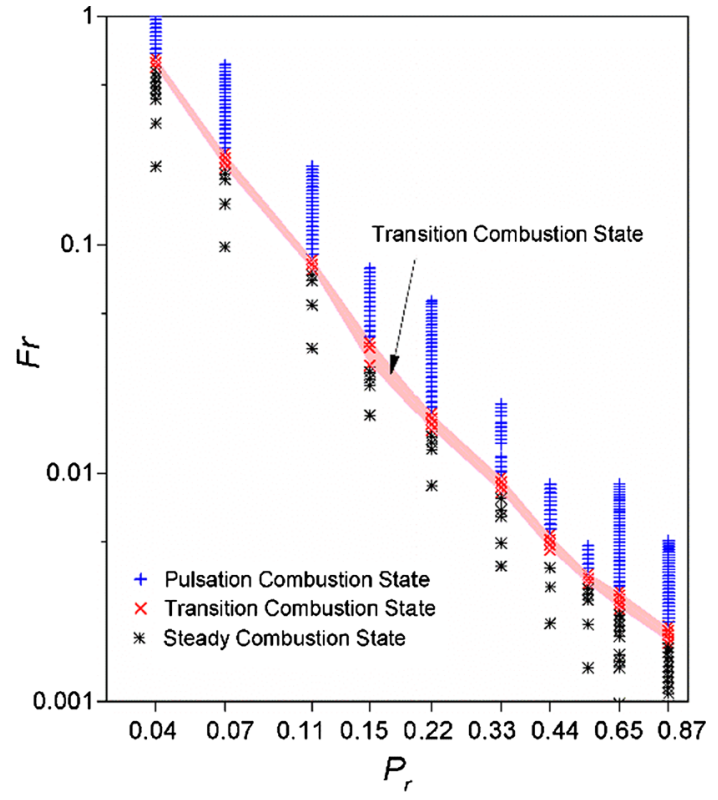

Fig. 12. Combustion states distribution at different combustion conditions.

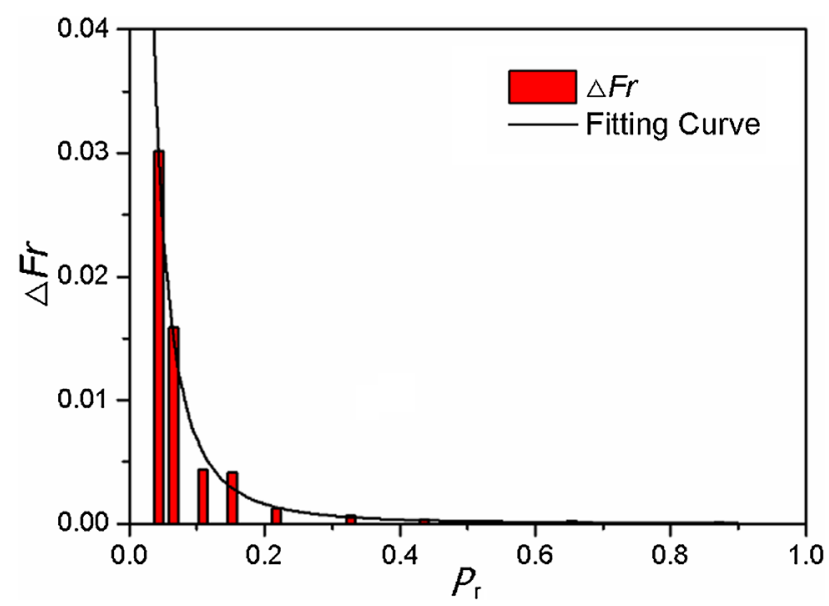

Fig. 13. Halfwidth variation of the transition combustion state at different pressures.

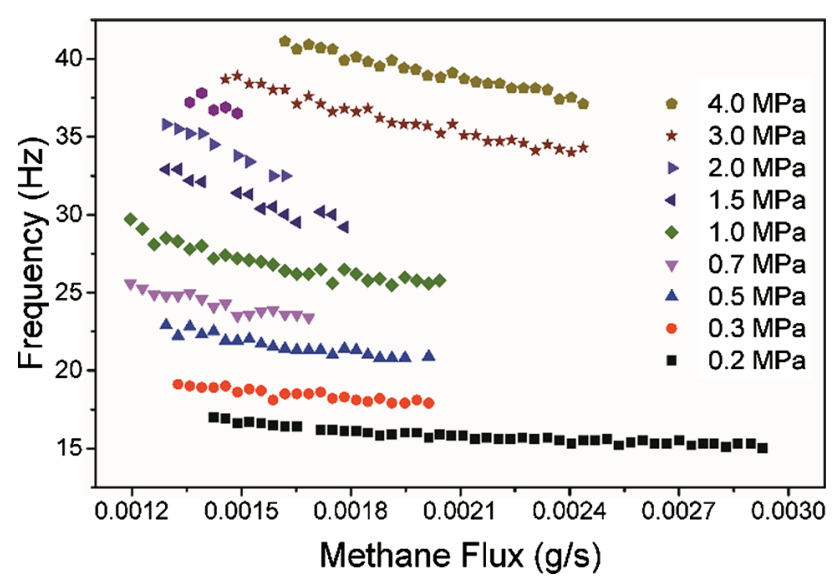

Fig. 14. Flame pulsation frequency in pulsation combustion state.

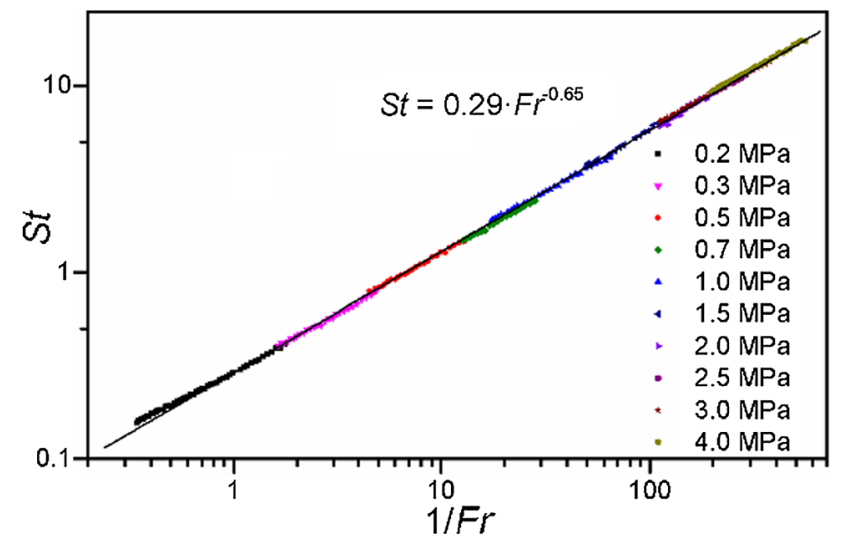

Fig. 15. Pulsation data fitting curve.

\subsection{Zone division of combustion states}

The buoyancy is primarily associated with the flames outside the fuel jet mixing layer, which significantly influences the combustion state of the laminar co-flow diffusion flame. Since the Froude number and pressure are related to the buoyancy, additional experiments were performed to investigate the effects of the Froude number and pressure on the flame combustion state.

Based on the flame luminous intensity variation with time for $F r=5 \times 10^{-4}$ to 3 and $p=0.2-4.0 \mathrm{MPa}$, the combustion states of the methane/oxygen diffusion flame are distinguished by the STFT method (see Fig. 7), and the zone division of the flame combustion states is shown in Fig. 12, where the relative pressure $p_{\mathrm{r}}$ is the ratio of the combustion pressure to the fuel critical pressure (for methane, the critical pressure is $4.59 \mathrm{MPa}$ ). As seen from Fig. 12, in the logarithmic coordinate system, the flame transition state is in a belt zone. With the increase in the combustion pressure, the corresponding Froude number decreases for the transition combustion state, the area of the pulsation combustion state increases, and the area of the steady combustion state decreases.

In the transition combustion state, random flame vibration occurs, which is harmful to the combustion devices in numerous practical applications; therefore, identifying the specific condition at which the transition combustion state appears is important for practical device design. Fig. 13 shows the half width $\triangle F r$ variation of the transition state zone shown in Fig. 12. With the increasing in combustion pressure, density gradient increases, leading to larger velocity gradient near the flame shear layer. Flame turns to be more sensitive, and easier to lose stability. Thus, the width of transition combustion state decreases with increasing combustion pressure. As a result, the increasing in the combustion pressure leads to sharply decrease in $\triangle F r$ at $p_{\mathrm{r}}<0.1$, and $\triangle F r$ approaches zero at $p_{\mathrm{r}}>0.5$.

In Fig. 12, the centre line of the Froude number $\left(F r_{\text {cent }}\right)$ of the transition state zone is fitted by:

$F r_{\text {cent }}=9.8 \times 10^{-4}\left(p_{\mathrm{r}}-0.012\right)^{-1.87}$

In Fig. 13, the half width $\triangle F r$ of the transition state is fitted by:

$F r=4.0 \times 10^{-5}\left(p_{\mathrm{r}}+0.026\right)^{-2.48}$

Thus, the upper and lower limits of the Froude number for the transition combustion state zone are as follows:

$\left\{\begin{array}{l}F r_{\text {crmax }}=F r_{\text {cent }}+\Delta F r \\ F r_{\text {crmin }}=F r_{\text {cent }}-\Delta F r\end{array}\right.$

where $F r_{\text {crmax }}$ and $F r_{\text {crmin }}$ are the upper and lower limits, respectively. At a given combustion pressure, the flame is in the steady combustion 
state at $F r<F r_{\text {crmin; }}$ the flame is in the pulsation combustion state at $F r>F r_{\text {crmax }}$; and, at $F r_{\text {crmin }}<F r<F r_{\text {crmax }}$, the flame is in the transition combustion state.

\subsection{Flame pulsation frequency variation in the pulsation combustion state}

In the pulsation combustion state, the flame pulsation frequencies at different combustion conditions is shown in Fig. 14. The flame pulsation frequency increases with combustion pressure, while decreases with methane flux.

To further investigate the effects of the combustion pressure and methane flux on the flame pulsation frequency, Froude number $(F r)$ and the dimensionless frequency Strouhal number $(S t)$ is employed. Strouhal number is defined as:

$S t=\frac{f_{t}}{l \cdot u}$

where $f_{t}$ is the flame pulsation frequency $(\mathrm{Hz}) ; u$ is the average methane flow speed at the burner port, $\mathrm{m} / \mathrm{s} ; g$ is the acceleration due to gravity, $\mathrm{m} / \mathrm{s}^{2}$; and $l$ is the characteristic length (here, $l$ is the diameter of burner port), $\mathrm{m}$.

The relation between $S t$ and $F r$ at $p=0.2-4.0 \mathrm{MPa}$ is shown in Fig. 15. In logarithmic coordinates, all data points show a linear relation between $S t$ and 1/Fr. While it appears that $S t$ is independent of the combustion pressure, the pressure actually influences the velocity of the laminar co-flow methane/oxygen jet flow, which finally results in the variations of Fr and St (see Eqs. (1) and (6)). Therefore, the dimensionless function relating the Strouhal number and Froude number may completely represent the behaviour of the flame pulsation frequency in pulsation combustion. With increasing $F r$, the inertia force of the jet flow increases, and the Kelvin-Helmholtz instability induced by buoyancy is weakened, thus increasing the Froude number of the laminar co-flowing injection flow that leads to the flame pulsation frequency decrease (Fig. 15).

The dimensionless function relation in Fig. 15 is fitted as:

$S t=0.29 \hat{\mathrm{A}} \cdot \mathrm{Fr}^{-0.65}$

Hamins et al. [26] presented the dimensionless function relation (StFr) for the laminar co-flowing methane/air diffusion combustion, with similar results to those described by Eq. (7), albeit with the exponent of -0.57 rather than -0.65 . These results indicate that the Froude number has a greater effect on the Strouhal number of the methane/air diffusion flame than that of the methane/oxygen diffusion flame because the nitrogen dilution effect should be taken into consideration when air is used as the oxidizer. Compared to the methane/air flame, oxygen diffuses into fuel much more easily in the methane/oxygen diffusion flame, and the flame height becomes shorter, weakening the effect of buoyancy on the flame so that the effect of Fr on St becomes weak in the methane/oxygen diffusion flame.

\section{Conclusion}

The stability characteristics of the laminar co-flow methane/oxygen diffusion flame with a $3 \mathrm{~mm}$ injector were investigated from $p=0.2$ to 4.0 MPa; the regions of the three flame combustion states were identified, the origins of the three combustion states were investigated by studying the flow field details captured using a Schlieren device, and the pulsation frequency characteristic of the flame was obtained. Some important conclusions were obtained as follows:

(1). The methane/oxygen diffusion flame has three combustion states: the steady combustion state, transition combustion state, and pulsation combustion state. These states are caused by the different formation heights of the vortices generated in the jet flame shear layer.

(2). The upper and lower limits of the Froude number for the transition combustion state zone at a given pressure are given by:

$\left\{\begin{array}{l}F r_{\text {crmax }}=F r_{\text {cent }}+\Delta F r \\ F r_{\text {crmin }}=F r_{\text {cent }}-\Delta F r\end{array}\right.$

where $F r_{\text {cent }}=9.8 \times 10^{-4}\left(p_{r}-0.012\right)^{-1.87}$ and $\triangle F r=4.0 \times$ $10^{-5}\left(p_{r}+0.026\right)^{-2.48}$.

(1). The flame is in the steady combustion state for $F r<F r_{\text {crmin }}$; the flame is in the pulsation combustion state for $F r>F r_{\text {crmax }}$; and the flame is in transition combustion state for $F r_{\text {crmin }}<F r<F r_{\text {crmax }}$.

(2). The dimensionless function relation between the Strouhal number and Froude number was found to be:

$S t=0.29 \hat{\mathrm{A}} \cdot F r^{-0.65}$

\section{Acknowledgements}

This work was supported by the National Natural Science Foundation of China (Grant No. 91641115) and the Strategic Priority Research Program of Chinese Academy of Sciences (Grant No. XDA17030100). The author greatly acknowledges Professor Xilong Yu of the Institute of Mechanics CAS for providing technical assistance in the present study.

\section{Appendix A. Supplementary data}

Supplementary data to this article can be found online at https:// doi.org/10.1016/j.fuel.2019.01.113.

\section{References}

[1] Rayleigh JW. The explanation of certain acoustic phenomena. Nature 1878;18(455):319-21. https://doi.org/10.1038/018319a0.

[2] Cammarata L, Fichera A, Pagano A. Neural prediction of combustion instability. Appl Energ 2002;72:513-28. (10.1016/S0306-2619(02)00024-7).

[3] Joo HI, Gülder ÖL. Soot formation and temperature structure in small methane-oxygen diffusion flames at subcritical and supercritical pressures. Combust Flame 2010;157(6):1194-201. https://doi.org/10.1016/j.combustflame.2009.11. 003.

[4] Liu F, Thomson KA, Guo H, Smallwood GJ. Numerical and experimental study of an axisymmetric coflow laminar methane-air diffusion flame at pressures between 5 and 40 atmospheres. Combust Flame 2006;146(3):456-71. https://doi.org/10. 1016/j.combustflame.2006.04.018.

[5] Li Sen, Wei Xiaolin. Skeletal mechanism modelling of n-heptane/oxygen laminar coflow flame structure at pressures. Fuel 2015;162(15):162-70. https://doi.org/10 1016/j.fuel.2015.09.013.

[6] Miller IM (NASA Langley Research Center, Hampton, VA), Maahs HG (NASA Langley Research Center, Hampton, VA). High pressure flame system for pollution studies with results for methane-air diffusion flames. Hampton (VA): NASA Langley Research Center (US), NASA Technical Note; 1977 June. Report No.: NASA-TN-D8407, L-11199. Contract No.: RTOP 505-03-31-01.

[7] Karatas AE, Intasopa G, Gülder ÖL. Sooting behavior of n-heptane laminar diffusion flames at high pressures. Combust Flame 2013;160(9):1650-6. https://doi.org/10 1016/j.combustflame. 2013.03.008.

[8] Kimura I. Stability of laminar-jet flames. Symp Combust 1965;10(1):1295-300. https://doi.org/10.1016/S0082-0784(65)80264-8.

[9] Durox D, Yuan T, Villermaux E. The effect of buoyancy on flickering in diffusion flames. Combust Sci Tech 1997;124(1-6):277-94. https://doi.org/10.1080/ 00102209708935648.

[10] Zhang D, Fang J, Guan JF, Wang JW, Zeng Y, Wang JJ, et al. Laminar jet methane/ air diffusion flame shapes and radiation of low air velocity coflow in microgravity. Fuel 2014;130(16):25-33. https://doi.org/10.1016/j.fuel.2014.04.008.

[11] Lewis GS, Cantwell BJ, Vandsburger U, Bowman CT. An investigation of the structure of a laminar non-premixed flame in an unsteady vortical flow. Symp Combust 1989;22(1):515-22. https://doi.org/10.1016/S0082-0784(89)80058-X.

[12] Charwath M, Hentschel J, Bockhorn H, Suntz R. Behavior of moderately oscillating sooting methane-air diffusion flames. Flow Turbulence Combust 2009;82(4):553. https://doi.org/10.1007/s10494-008-9197-4.

[13] Papadopoulos G, Bryant R, Pitts W. Flow characterization of flickering methane/air diffusion flames using particle image velocimetry. Exp Fluids 2002;33(3):472-81. https://doi.org/10.1007/s00348-002-0483-y.

[14] Buckmaster J, Peters N. The infinite candle and its stability - a paradigm for flickering diffusion flames. Symp Combust 1988;21(1):1829-36. https://doi.org/ 10.1016/S0082-0784(88)80417-X.

[15] Darabkhani HG, Bassi J, Huang HW, Zhang Y. Fuel effects on diffusion flames at elevated pressures. Fuel 2009;88(2):264-71. https://doi.org/10.1016/j.fuel.2008. 
09.013

[16] Hegde U, Zhou L, Bahadori MY. The transition to turbulence of microgravity gas jet diffusion flames. Combust Sci Tech 1994;102:95-113. https://doi.org/10.1080/ 00102209408935471.

[17] Maxworthy T. The flickering candle: transition to a global oscillation in a thermal plume. J Fluid Mech 1999;390:297-323. https://doi.org/10.1007/978-94-011 5118-4_2.

[18] Manikantachari KRV, Raghavan V, Srinivasan K. Natural flickering of methane diffusion flames. Int J Mech Mechatron Eng 2011;59(11). pp. 2423-8. (dai:10.1999/1307-6892/5875.

[19] Welch PD. The use of fast Fourier transform for the estimation of power spectra: a method based on time averaging over short, modified periodograms. IEEE Trans Audio Electroacoust 1967;15(2):70-3. https://doi.org/10.1109/TAU.1967. 1161901.

[20] Sejdić E, Djurović I, Jiang J. Time-frequency feature representation using energy concentration: an overview of recent advances. Digit Signal Process 2009;19(1):153-83. https://doi.org/10.1016/j.dsp.2007.12.004.

[21] Fan Y, Suzuk Y, Kasagi N. Quenching mechanism study of oscillating flame in micro channels using phase-locked OH-PLIF. Proc Combust Inst 2001;33(2):3267-73. https://doi.org/10.1016/j.proci.2010.05.041.

[22] H.G. Darabkhani Experimental investigations on sooty flames at elevated pressures 2010 University of Manchester (http://www.manchester.ac.uk/escholar/uk-ac man-scw:80292).

[23] H. Guo Flame and acoustic waves interactions and flame control 2011 University of Manchester (https://www.escholar.manchester.ac.uk/uk-ac-man-scw:123468).

[24] Gotoda H, Kawaguchi S, Saso Y. Experiments on dynamical motion of buoyancyinduced flame instability under different oxygen concentration in ambient gas. Exp Therm Fluid Sci 2008;32(8):1759-65. https://doi.org/10.1016/j.expthermflusci. 2008.05.005.

[25] Gotoda H, Ueda T, Shepherd IG, Cheng RK. Flame flickering frequency on a rotating Bunsen burner. Chem Eng Sci 2007;62(6):1753-9. https://doi.org/10.1016/j.ces. 2006.11.012.

[26] Hammis A, Yang JC, Kashiwagi T. An experimental investigation of the pulsation frequency of flames. Symp Combust 1992;24(1):1695-702. https://doi.org/10. 1016/S0082-0784(06)80198-0. 